\title{
Bias Reduction for the Maximum Likelihood Estimators of the Parameters in the Half-Logistic Distribution
}

\author{
David E. Giles \\ Department of Economics, University of Victoria \\ Victoria, B.C., Canada V8W $2 Y 2$
}

Revised, June 2010

\begin{abstract}
We derive analytic expressions for the biases of the maximum likelihood estimators of the scale parameter in the half-logistic distribution with known location, and of the location parameter when the latter is unknown. Using these expressions to bias-correct the estimators is highly effective, without adverse consequences for estimation mean squared error. The overall performance of the first of these bias-corrected estimators is slightly better than that of a bootstrap bias-corrected estimator. The bias-corrected estimator of the location parameter significantly out-performs its bootstrapped-based counterpart. Taking computational costs into account, the analytic bias corrections clearly dominate the use of the bootstrap.
\end{abstract}

\section{Keywords Half-logistic distribution; Life testing; Bias reduction}

Mathematics Subject Classification $\quad 62 \mathrm{~F} 10 ; 62 \mathrm{~F} 40 ; 62 \mathrm{~N} 02 ; 62 \mathrm{~N} 05$

\section{Author Contact:}

David E. Giles, Dept. of Economics, University of Victoria, P.O. Box 1700, STN CSC, Victoria, B.C., Canada V8W 2Y2; e-mail: dgiles@uvic.ca; Phone: (250) 721-8540; FAX: (250) 721-6214 


\section{Introduction}

The half-logistic (or folded-logistic) distribution was proposed by Balakrishnan (1985) as a lifetesting model. One of the attractions of this distribution in the context of reliability theory is that it has a monotonically increasing hazard rate for all parameter values, a property shared by relatively few distributions which have support on the positive real half-line. In terms of tail behaviour, the half-logistic distribution provides a degree of flexibility as its tail thickness lies between those of the half-normal and half-Cauchy distributions. The half-logistic distribution has

also been used successfully to model records. For example, Mbah and Tsokos (2008) apply it to environmental and sports records data.

If $X$ follows a logistic distribution, then $Y=|X|$ has a half-logistic distribution, the p.d.f. for which is:

$$
\begin{aligned}
f(y) & =\frac{(2 / \sigma) \exp \{-(y-\mu) / \sigma\}}{[1+\exp \{-(y-\mu) / \sigma\}]^{2}} \\
& =\frac{(2 / \sigma) \exp \{(y-\mu) / \sigma\}}{[1+\exp \{(y-\mu) / \sigma\}]^{2}}
\end{aligned} ; \quad y \geq \mu \geq 0, \sigma>0
$$

where $\mu$ and $\sigma$ are the location and scale parameters respectively. The moments of this distribution are given in Appendix A.1.

Various estimators for the parameters of the half-logistic distribution have been proposed, for both uncensored and censored data. For example, see Balakrishnan and Puthenpura (1986), Balakrishnan and Wong (1991), Balakrishnan and Chan (1992), and Adatia (1997, 2000). In addition, the operating characteristic under acceptance sampling from the half-logistic distribution has been discussed by Kantam and Rosaiah (1998). In this paper we deal with maximum likelihood estimation with uncensored data.

If the location parameter of (1) is unknown, its MLE is well-known to be the smallest order statistic in the sample (e.g., Balakrishnan and Wong, 1991, p.142). However, the MLE for the scale parameter cannot be expressed in closed form, whether the location parameter is known or not. Notwithstanding this complication, we derive the bias, to $O\left(n^{-1}\right)$, of the MLE of the scale parameter of the half-logistic distribution in the interesting case where the location parameter is known to be zero. It transpires that this MLE has extremely small relative bias, even in very small samples. We also consider a very simple bias-corrected counterpart to this estimator, and show that its bias is an order of magnitude less than that of the MLE itself, and that this is achieved 
without increasing relative mean squared error (MSE). The alternative approach of using the bootstrap to correct for the bias of the MLE is found to be inferior to our analytic correction.

We also simulate the bias and MSE of the MLE's of the scale and location parameters, and their bootstrap-bias-adjusted counterparts, when the location parameter is unknown. In this case exact expressions for the first two moments of the MLE for the location parameter can be deduced from the recurrence formulae given by Balakrishnan (1985) for the standardized half-logistic distribution. In particular, the associated exact expression for the bias of the MLE of the location parameter depends only on the sample size and the true value of the scale parameter. So, biascorrecting the MLE for the location parameter is quite straightforward in this case, and we show that it is highly effective. As noted above, when the location parameter is unknown the MLE for the scale parameter cannot be expressed in closed form. Unfortunately, the methods that we adopt to obtain the $O\left(n^{-1}\right)$ bias of this MLE in the case of a known location parameter cannot be used when this parameter is unknown. A bootstrap-based bias correction is also considered in this case, but it has little impact on the performance of the MLE of the scale parameter.

The next section presents summarizes the analytic techniques that we use to evaluate the bias of the MLE of the scale parameter, to $O\left(n^{-1}\right)$, when the location parameter is known. Our principal results appear in section 3; and a selection of numerical evaluations are given in section 4. Section 5 concludes. Some technical details are provided in Appendix A.

\section{Preliminary Results}

Let $l(\theta)$ be the log-likelihood function based on a sample of $n$ observations, with $p$-dimensional parameter vector, $\theta \cdot l(\theta)$ is assumed to be regular with respect to all derivatives up to and including the third order. Define:

$$
\begin{aligned}
& k_{i j}=E\left(\partial^{2} l / \partial \theta_{i} \partial \theta_{j}\right) \quad ; \quad i, j=1,2, \ldots, p \\
& k_{i j l}=E\left(\partial^{3} l / \partial \theta_{i} \partial \theta_{j} \partial \theta_{l}\right) \quad ; \quad i, j, l=1,2, \ldots, p \\
& k_{i j, l}=E\left[\left(\partial^{2} l / \partial \theta_{i} \partial \theta_{j}\right)\left(\partial l / \partial \theta_{l}\right)\right] ; \quad i, j, l=1,2, \ldots, p .
\end{aligned}
$$

and

$$
k_{i j}^{(l)}=\partial k_{i j} / \partial \theta_{l} \quad ; \quad i, j, l=1,2, \ldots, p .
$$

All of the expressions in (2) - (5) are assumed to be $O(n)$. Extending earlier work by Tukey (1949), Bartlett (1953a, 1953b), Haldane (1953), Haldane and Smith (1956), Shenton and 
Wallington (1962) and Shenton and Bowman (1963), Cox and Snell (1968) showed that when the sample data are independent (but not necessarily identically distributed) the bias of the $s^{\text {th }}$ element of the MLE of $\theta(\hat{\theta})$ is:

$$
\operatorname{Bias}\left(\hat{\theta}_{s}\right)=\sum_{i=1}^{p} \sum_{j=1}^{p} \sum_{l=1}^{p} k^{s i} k^{j l}\left[0.5 k_{i j l}+k_{i j, l}\right]+O\left(n^{-2}\right) ; \quad s=1,2, \ldots ., p .
$$

where $k^{i j}$ is the $(i, j)^{\text {th }}$ element of the inverse of the (expected) information matrix, $K=\left\{-k_{i j}\right\}$. Cordeiro and Klein (1994) note that this bias expression also holds if the data are nonindependent, provided that all of the $k$ terms are $O(n)$, and show that it can be re-written as:

$$
\operatorname{Bias}\left(\hat{\theta}_{s}\right)=\sum_{i=1}^{p} k^{s i} \sum_{j=1}^{p} \sum_{l=1}^{p}\left[k_{i j}^{(l)}-0.5 k_{i j l}\right] k^{j l}+O\left(n^{-2}\right) ; s=1,2, \ldots ., p .
$$

The computational advantage of (7) over (6) is that it does not involve terms of the form defined in (4).

Now, let $a_{i j}^{(l)}=k_{i j}^{(l)}-\left(k_{i j l} / 2\right)$, for $i, j, l=1,2, \ldots, p$; and define the following matrices:

$$
\begin{aligned}
& A^{(l)}=\left\{a_{i j}^{l}\right\} ; \quad i, j, l=1,2, \ldots ., p \\
& A=\left[A^{(1)}\left|A^{(2)}\right| \ldots \ldots . . \mid A^{(p)}\right] .
\end{aligned}
$$

Cordeiro and Klein (1994) show that the expression for the $O\left(n^{-1}\right)$ bias of $\hat{\theta}$ can be re-written as:

$$
\operatorname{Bias}(\hat{\theta})=K^{-1} A \operatorname{vec}\left(K^{-1}\right)+O\left(n^{-2}\right)
$$

A “bias-corrected" MLE for $\theta$ can then be obtained as:

$$
\tilde{\theta}=\hat{\theta}-\hat{K}^{-1} \hat{A} \operatorname{vec}\left(\hat{K}^{-1}\right),
$$

where $\hat{K}=\left.(K)\right|_{\hat{\theta}}$ and $\hat{A}=\left.(A)\right|_{\hat{\theta}}$, and it can be shown that the bias of $\tilde{\theta}$ will be $O\left(n^{-2}\right)$. Unfortunately, the Cox-Snell methodology does not facilitate the derivation of the variance of the MLE for $\theta$ or for $\tilde{\theta}$. Finally, it is crucial to note that (10) and (11) can still be evaluated even when the likelihood equation does not admit a closed-form analytic solution, and the MLE has to be obtained numerically. 


\section{Bias of the MLE}

\subsection{Known location parameter}

Under independent sampling from the half-logistic distribution, with uncensored data, the log-likelihood function when $\mu=0$ is:

$$
l=n \ln (2)-n \ln (\sigma)+(n \bar{y} / \sigma)-2 \sum_{i=1}^{n} \ln \left[1+\exp \left(y_{i} / \sigma\right)\right]
$$

where $\bar{y}=n^{-1} \sum_{i=1}^{n} y_{i}$. So,

$$
\partial l / \partial \sigma=-(n / \sigma)-\left(n \bar{y} / \sigma^{2}\right)+\left(2 / \sigma^{2}\right) \sum_{i=1}^{n}\left[y_{i} \exp \left(y_{i} / \sigma\right)\right] /\left[1+\exp \left(y_{i} / \sigma\right)\right] \quad .
$$

Note that there is no closed-form solution to the likelihood equation obtained by equating (13) to zero.

In what follows, we will require the following derivatives of the log-likelihood function:

$$
\begin{aligned}
\partial^{2} l / \partial \sigma^{2}= & \left(n / \sigma^{2}\right)+\left(2 n \bar{y} / \sigma^{3}\right)-\left(4 / \sigma^{3}\right) \sum_{i=1}^{n}\left[y_{i} \exp \left(y_{i} / \sigma\right)\right] /\left[1+\exp \left(y_{i} / \sigma\right)\right] \\
& -\left(2 / \sigma^{4}\right) \sum_{i=1}^{n}\left[y_{i}^{2} \exp \left(y_{i} / \sigma\right)\right] /\left[1+\exp \left(y_{i} / \sigma\right)\right]^{2} \\
\partial^{3} l / \partial \sigma^{3}= & -\left(2 n / \sigma^{3}\right)-\left(6 n \bar{y} / \sigma^{4}\right)+\left(12 / \sigma^{4}\right) \sum_{i=1}^{n}\left[y_{i} \exp \left(y_{i} / \sigma\right)\right] /\left[1+\exp \left(y_{i} / \sigma\right)\right] \\
+ & \left(12 / \sigma^{5}\right) \sum_{i=1 i}^{n}\left[y_{i}^{2} \exp \left(y_{i} / \sigma\right)\right] /\left[1+\exp \left(y_{i} / \sigma\right)\right]^{2} \\
+ & \left(2 / \sigma^{6}\right) \sum_{i=1 i}^{n}\left[y_{i}^{3}\left\{\exp \left(y_{i} / \sigma\right)-\exp \left(2 y_{i} / \sigma\right)\right\}\right] /\left[1+\exp \left(y_{i} / \sigma\right)\right]^{3}
\end{aligned}
$$

To evaluate the expectations of these derivatives we will use the following results for a halflogistic variate, $Y$, the proofs of which appear in Appendix A.2:

$$
\begin{aligned}
& E\{[y \exp (y / \sigma)] /[1+\exp (y / \sigma)]\}=\sigma[\ln (2)+0.5] \\
& E\left\{\left[y^{2} \exp (y / \sigma)\right] /[1+\exp (y / \sigma)]^{2}\right\}=\left(\sigma^{2} / 3\right)\left[\left(\pi^{2} / 6\right)-1\right] \\
& E\left\{\left[y^{3}(\exp (y / \sigma)-\exp (2 y / \sigma))\right] /[1+\exp (y / \sigma)]^{3}\right\}=\sigma^{3}\left[0.5-\left(\pi^{2} / 12\right)\right]
\end{aligned}
$$

We then have the following expressions relating to the joint cumulants of the derivatives of the log-likelihood function: 


$$
\begin{aligned}
k_{11} & =E\left[\partial^{2} l / \partial \sigma^{2}\right]=-\left(n / 9 \sigma^{2}\right)\left(3+\pi^{2}\right) \\
& =-1.429956044\left(n / \sigma^{2}\right) \\
k_{111} & =E\left[\partial^{3} l / \partial \sigma^{3}\right]=\left(n / 2 \sigma^{3}\right)\left(2+\pi^{2}\right) \\
& =5.934802199\left(n / \sigma^{3}\right)
\end{aligned}
$$

In addition,

$$
\begin{aligned}
k_{11}^{(1)} & =\partial k_{11} / \partial \sigma=2 n\left(3+\pi^{2}\right) /\left(9 \sigma^{3}\right) \\
& =2.859912088\left(n / \sigma^{3}\right)
\end{aligned}
$$

and

$$
\begin{aligned}
a_{11}^{(1)} & =k_{11}^{(1)}-0.5 k_{111} \\
& =-0.107489011\left(n / \sigma^{3}\right)
\end{aligned}
$$

The (expected) information measure is

$$
K=-k_{11}=1.429956044\left(n / \sigma^{2}\right) \quad,
$$

and

$$
A=a_{11}^{(1)} \quad \text {. }
$$

So, using Cordeiro and Klein's (1994) modification of the Cox-Snell (1968) result, to $O\left(n^{-1}\right)$,

$$
\begin{aligned}
\operatorname{Bias}(\hat{\sigma}) & =K^{-1} \operatorname{Avec}\left(K^{-1}\right) \\
& =-0.052567665(\sigma / n)
\end{aligned}
$$

The bias is unambiguously negative, and very small in relative terms. Of course, this is a very encouraging result for practitioners who apply this distribution. Moreover, the relative bias is invariant to the value of the scale parameter. An unbiased (to $O\left(n^{-2}\right)$ ) estimator of $\sigma$ can be constructed as $\tilde{\sigma}=(\hat{\sigma}-\operatorname{Bias}(\hat{\sigma}))=\hat{\sigma}(n+0.052567665) / n$. Correcting for the bias in this way also has implications for the MSE of the estimator, and this point is taken up in section 4.

\subsection{Unknown location}

In this case the log-likelihood function is

$$
l=n \ln (2)-n \ln (\sigma)+(n \bar{y} / \sigma)-(n \mu / \sigma)-2 \sum_{i=1}^{n} \ln \left[1+\exp \left\{\left(y_{i}-\mu\right) / \sigma\right\}\right] .
$$

The MLE for $\mu$ is $\hat{\mu}=y_{1: n}$, the smallest sample order statistic. Balakrishnan (1985) provides values for the mean of $\hat{\mu}$ for the standardized half-logistic variate, $Z$. Immediately, it follows that $\operatorname{Bias}(\hat{\mu})=\sigma E(Z)$, where $E(Z)$ can be derived from the recurrence formula (2.2) in Balakrishnan 
(1985, p.290), and is tabulated as the numerical quantity $\alpha_{1: n}$ in his Table I, for small values of $n$.

A bias-corrected estimator of $\mu$ can then be constructed as $\tilde{\mu}=\hat{\mu}-\hat{\sigma} \alpha_{1: n}$, where $\hat{\sigma}$ is the MLE for $\sigma$ obtained by solving equation (28) below, numerically.

Now consider the estimation of $\sigma$. Using the expression for $\hat{\mu}$, the profile log-likelihood function is

$$
l^{*}=n \ln (2)-n \ln (\sigma)+(n \bar{y} / \sigma)-\left(n y_{1: n} / \sigma\right)-2 \sum_{i=1}^{n} \ln \left[1+\exp \left\{\left(y_{i}-y_{1: n}\right) / \sigma\right\}\right]
$$

and the likelihood equation for the scale parameter is

$$
\begin{aligned}
\partial l^{*} / \partial \sigma= & -(n / \sigma)-\left(n \bar{y} / \sigma^{2}\right)+\left(n y_{1: n} / \sigma^{2}\right) \\
& +\left(2 / \sigma^{2}\right) \sum_{i=1}^{n}\left(\left[\left(y_{i}-y_{1: n}\right) \exp \left\{\left(y_{i}-y_{1: n}\right) / \sigma\right\}\right] /\left[1+\exp \left\{\left(y_{i}-y_{1: n}\right) / \sigma\right\}\right]\right)=0
\end{aligned}
$$

Equation (28) does not admit a closed-form solution for $\sigma$ so the MLE, $\hat{\sigma}$, must be obtained numerically. Moreover, to see that the Cox-Snell methodology adopted in section 3.1 is not feasible in this case, it suffices to consider the second derivative of the log-likelihood equation:

$$
\begin{aligned}
\partial^{2} l^{*} / \partial \sigma^{2} & =\left(n / \sigma^{2}\right)+2\left(n \bar{y} / \sigma^{3}\right)-2\left(n y_{1: n} / \sigma^{3}\right) \\
& -\left(4 / \sigma^{3}\right) \sum_{i=1}^{n}\left[\left(y_{i}-y_{1: n}\right) \exp \left\{\left(y_{i}-y_{1: n}\right) / \sigma\right\}\right] /\left[1+\exp \left\{\left(y_{i}-y_{1: n}\right) / \sigma\right\}\right] \\
& -\left(2 / \sigma^{4}\right) \sum_{i=1}^{n}\left[\left(y_{i}-y_{1: n}\right)^{2} \exp \left\{\left(y_{i}-y_{1: n}\right) / \sigma\right\}\right] /\left[1+\exp \left\{\left(y_{i}-y_{1: n}\right) / \sigma\right\}\right]
\end{aligned}
$$

To evaluate the expectation of the expression in (29) requires knowledge of the joint distribution of $y_{i}$ and $y_{1: n}$, which does not appear to be tractable. The situation becomes even more complicated when the higher order derivatives are considered. So, we are unable to use the CoxSnell approach to obtain an expression for the $O\left(n^{-1}\right)$ bias of $\hat{\sigma}$ in this case.

\section{Numerical Evaluations}

The biases and MSEs of the various maximum likelihood and bias-corrected maximum likelihood estimators have been simulated in a Monte Carlo experiment. The simulations were undertaken using the maxLik package (Toomet, 2008) for the $R$ statistical software environment (R, 2008), on a P.C. with a Pentium 4 processor clocked at $2 \mathrm{GHz}$ running Windows XP-Pro. Each part of the experiment uses 20,000 Monte Carlo replications. Half-logistic variates were generated using the 
inversion method, and the log-likelihood function was maximized using the Nelder-Mead algorithm and the results were verified with the (slower) Newton-Raphson algorithm.

Bootstrap-bias-corrected estimators are also investigated as obvious competitors to the analytic bias-corrected estimators that we are considering. The bootstrap-bias-corrected estimator of $\sigma$, for example, is defined as $\breve{\sigma}=2 \hat{\sigma}-\left(1 / N_{B}\right)\left[\sum_{j=1}^{N_{B}} \hat{\sigma}_{(j)}\right]$, where $\hat{\sigma}_{(j)}$ is the MLE of $\sigma$ obtained from the $j^{\text {th }}$ of the $N_{B}$ bootstrap samples. See Efron (1982, p.33). This estimator is also unbiased to $O\left(n^{-2}\right)$, but in many applications it is known to suffer from inflated variance. We have assigned $N_{B}=1,000$. Bias correction based on the bootstrap is the only method for reducing the bias of the MLE of $\sigma$ that we have considered when the location parameter is unknown, for the reasons outlined above.

\subsection{Known location}

The expression in (25), for the bias of $\hat{\sigma}$ when $\mu$ is known, is valid only to $O\left(n^{-1}\right)$. The actual bias of this estimator is illustrated in Table 1. There, we report simulated percentage biases and MSE's, the former defined as $100 \times\left(\right.$ Bias / $\sigma$ ) and the latter defined as $100 \times\left(\mathrm{MSE} / \sigma^{2}\right)$. For each of the estimators under consideration in Table 1, both of these measures are invariant to the value of $\sigma$, for a given sample size.

Several key results emerge from Table 1. First, the percentage bias of the MLE is negative but extremely small, even for very small sample sizes, which is most encouraging for users of this estimator. Second, however, the absolute bias of the bias-corrected estimator, $\tilde{\sigma}$, is often an order of magnitude less than that of the original MLE. Although there may be limited motivation for using this bias-corrected estimator, it is trivial to implement, and its bias is negligible, in percentage terms. Third, these gains in bias reduction when using $\tilde{\sigma}$ come at the cost of increases in variance, as is evidenced by the very small differences in the percentage MSE's that are reported for $\hat{\sigma}$ and $\tilde{\sigma}$. Fourth, the bootstrap-bias-corrected estimator performs at least as well as $\tilde{\sigma}$, in terms of (absolute) bias reduction when $n<20$, but at considerable additional computational expense. The cpu time taken to obtain $\hat{\sigma}$ and $\tilde{\sigma}$ is between 0.02 and 0.03 seconds for the sample sizes in Table 1 . In contrast, obtaining $\hat{\sigma}$ and the bootstrap-corrected estimator, $\breve{\sigma}$, takes between 10.3 and 10.4 seconds of cpu time. 
Finally, the bootstrap correction is also slightly less effective than the analytic correction in terms of the resulting relative mean squared error. Overall, taking into account the relative computational costs, these results support using the Cox-Snell analytic approach to correct for bias to $O\left(n^{-1}\right)$.

\subsection{Unknown location}

Table 2 reports the simulation results for the case where $\mu$ is unknown. It will be recalled that the MLE for $\mu(\hat{\mu})$ is the smallest order statistic in the sample. The properties of $\hat{\mu}$ and the analytically bias-adjusted estimator, $\tilde{\mu}$, proposed in section 3.2 , are compared with those of the bootstrap-bias-corrected estimator. The results for $\hat{\mu}$ (but not $\hat{\sigma}$ ) can be computed exactly from the information in Tables I and II of Balakrishnan (1985) for $n \leq 15$, and from our own extensions to these tables (available at http:web.uvic.ca/ dgiles/downloads/half-logistic) for $n>15$. These values for the relative bias and MSE of $\hat{\mu}$ were also used to verify that the simulation results in Table 2 are accurate to the reported precision. As it is not feasible to develop an analytic bias correction for $\hat{\sigma}$ in this case, the properties of this MLE are compared only with those of its bootstrap-bias-corrected counterpart. For a given sample size, all of the results for the estimators of $\sigma$ in Table 2 are invariant to the values of $\sigma$ and $\mu$, which are set to $\sigma=1$ and $\mu=$ 10. If we multiply $\mu$ ( $\sigma)$ by a constant, $c(d)$, this scales the relative biases and MSE's for the estimators of $\mu$ in Table 2 by $c^{-1}$ and $c^{-2}\left(d\right.$ and $\left.d^{-1}\right)$ respectively.

The MLE of the scale parameter, $\hat{\sigma}$, in Table 2 is again biased downwards, but the relative bias is approximately two orders of magnitude greater than in the case where $\mu$ is known. The bootstrap bias correction offers only a marginal improvement in terms of either bias or MSE. The MLE of the location parameter is positively biased, and this bias can be approximately halved (with a slight reduction in MSE) by implementing a bootstrap bias correction. The analytic biascorrected estimator, $\tilde{\mu}$, has negligible bias and even smaller MSE than its bootstrap-corrected counterpart. The excellent performance of $\tilde{\mu}$ is not surprising as the associated analytic bias correction would be exact (for all $n$ ) if $\sigma$ were known. This corrected estimator is more effective and more cost-efficient than the bootstrap-corrected estimator of the location parameter. The computing time taken to obtain $\hat{\sigma}, \hat{\mu}$ and $\tilde{\mu}$ is between 0.02 and 0.03 seconds for the sample sizes in Table 2. On the other hand, obtaining $\hat{\sigma}, \hat{\mu}$ and the bootstrap-bias-corrected estimators ( $\breve{\sigma}$ and $\breve{\mu}$ ) takes between 18.2 and 19.8 seconds. 


\section{Conclusion}

The maximum likelihood estimator of the scale parameter in the half-logistic distribution with a known location is found to have an extremely small (negative) percentage bias, even with quite small sample sizes. Using the Cox-Snell procedure for determining the $O\left(n^{-1}\right)$ bias of this estimator, and then making the associated analytic bias correction, reduces this small bias by a further order of magnitude. This is achieved without additional MSE, and the computational cost of bias adjusting the MLE is negligible. Similar results can be obtained using a bootstrap biascorrected estimator, but with substantially greater computational cost.

When the location parameter of the half-logistic distribution is unknown, the MLE of the scale parameter exhibits marked negative percentage bias, and this cannot be reduced to any degree by bootstrap bias-correcting the estimator. In contrast, the positive relative bias of the MLE of the location parameter can be approximately halved by using a bootstrap bias correction, and almost eliminated by using a simple analytic correction that is both computationally trivial and also reduces the relative MSE of the estimator.

In summary, the various analytic bias corrections that are explored in this paper either outperform, or are as effective as, the corresponding bootstrap bias corrections. When the relative computational costs are taken into account, the analytic corrections clearly dominate.

\section{Acknowledgment}

The author is grateful to Helen Feng and Ryan Godwin for helpful discussions and suggestions, and to two referees for their constructive comments on an earlier version of this paper. 


\section{Table 1}

Simulated percentage biases and mean squared errors: known location parameter

\begin{tabular}{lllcccc}
$n$ & $\% \operatorname{Bias}(\hat{\sigma})$ & $\% \operatorname{Bias}(\tilde{\sigma})$ & $\% \operatorname{Bias}(\tilde{\sigma})$ & $\% \operatorname{MSE}(\hat{\sigma})$ & $\% \operatorname{MSE}(\tilde{\sigma})$ & $\% \operatorname{MSE}(\tilde{\sigma})$ \\
\hline 10 & -0.675 & -0.153 & 0.103 & 6.875 & 6.943 & 6.991 \\
15 & -0.370 & -0.021 & 0.014 & 4.561 & 4.591 & 4.644 \\
20 & -0.295 & -0.033 & 0.042 & 3.472 & 3.490 & 3.524 \\
25 & -0.207 & -0.003 & 0.102 & 2.776 & 2.787 & 2.823 \\
50 & -0.123 & -0.018 & 0.098 & 1.405 & 1.407 & 1.423 \\
75 & -0.114 & -0.044 & 0.097 & 0.935 & 0.936 & 0.931 \\
100 & -0.062 & -0.009 & 0.031 & 0.704 & 0.705 & 0.711 \\
\hline
\end{tabular}

Table 2

Simulated percentage biases and mean squared errors: unknown location parameter

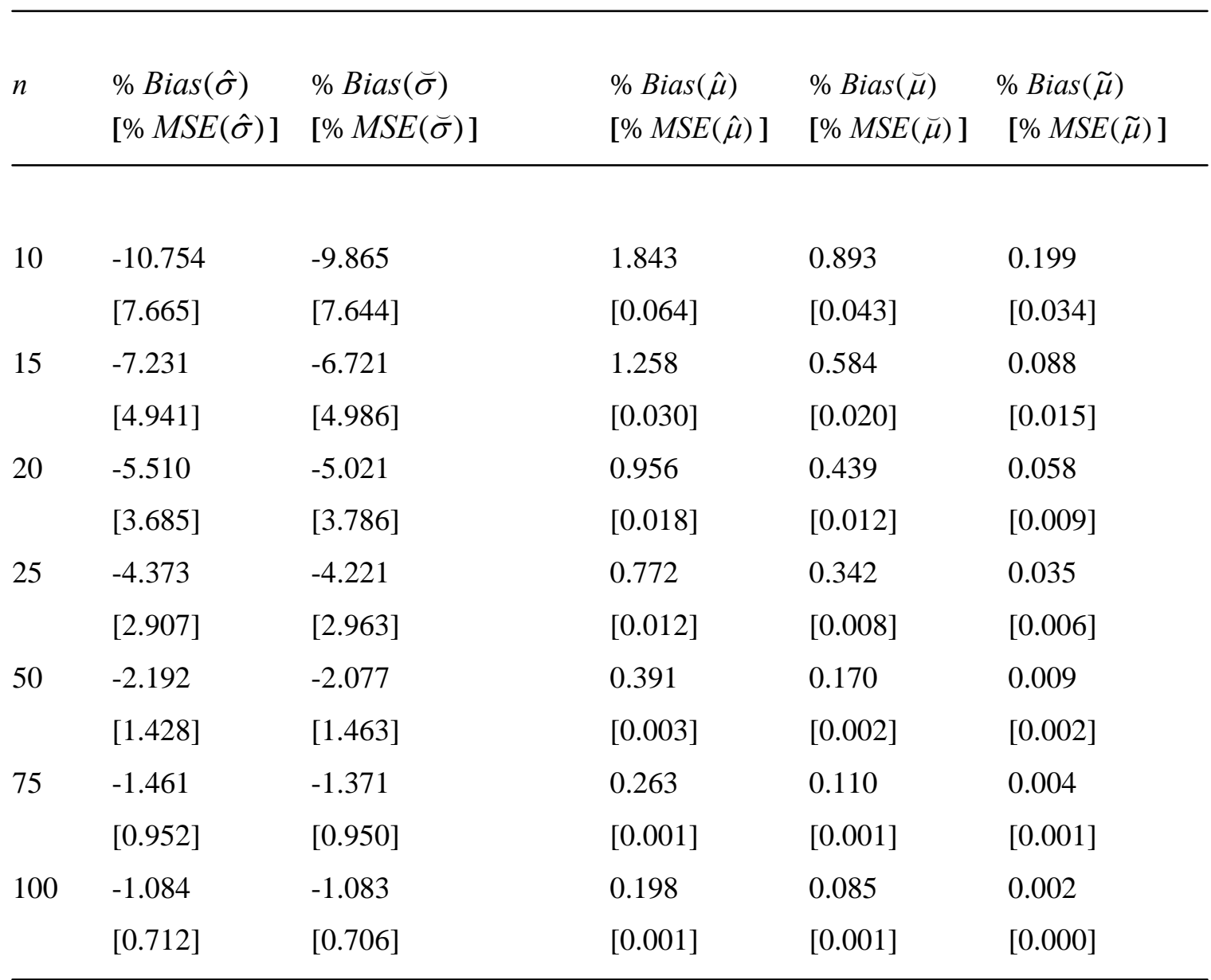




\section{Appendix A}

\section{A.1 Moments of the half-logistic distribution}

The central moments of the standardized half-logistic distribution can be evaluated using the following result (Gradshteyn, and Ryzhik, 1994; integral 3.424, no.2):

$$
\int_{0}^{\infty} \frac{(1+a) e^{x}+a}{\left(1+e^{x}\right)^{2}} e^{-a x} x^{n} d x=n ! \sum_{k=0}^{\infty} \frac{(-1)^{k}}{(a+k+1)^{n}}
$$

Letting $Z=(Y-\mu) / \sigma$, from (1) the density of the standardized variate, $Z$, is

$$
f(z)=\frac{2 e^{z}}{\left[1+e^{z}\right]^{2}} \quad ; \quad z>0
$$

and

$$
E\left(Z^{r}\right)=2 \int_{0}^{\infty} \frac{x^{r} e^{z}}{\left[1+e^{z}\right]^{2}} d z
$$

Applying (A.1) with $a=0$ and $n=r$ :

$$
E\left(Z^{r}\right)=2 r ! \sum_{k=0}^{\infty}(-1)^{k}(k+1)^{-r} .
$$

An alternative derivation of (A.2) is provided by Balakrishnan and Wong (1991, p. 140). Using the Maclaurin series for $\ln (1+w)$ with $w=1$, it follows that $E(Z)=\ln (4)$. Similarly, using the relationship $\left(\pi^{2} / 6\right)=\sum_{k=1}^{\infty} k^{-2}$, we have $E\left(Z^{2}\right)=\left(\pi^{2} / 3\right)$. The moments of $Y$ itself can, of course, be derived directly from (A.2) by applying the binomial theorem:

$$
E\left(Y^{r}\right)=2 r ! \sigma^{r} \sum_{j=0}^{r}{ }^{r} C_{j}(\mu / \sigma)^{r-j} \sum_{k=0}^{\infty}(-1)^{k}(k+1)^{-j} .
$$

When $\mu=0$, using the convention that $0^{0}=1$, it follows immediately that $E(Y)=\sigma \ln (4)$ and $V(Y)=\sigma^{2}\left\{\left(\pi^{2} / 3\right)-4[\ln (2)]^{2}\right\}$, etc. 


\section{A.2 Derivation of equations (16) - (18)}

All of the following results have been established analytically, and then verified by using the Maple 10 package (Maplesoft, 2005). Further details are available on request to the author.

(i) Equation (16) follows directly from (13) by recalling that $E(\partial l / \partial \sigma)=0$, and using the result that $E(Y)=2 \sigma \ln (2)$, from Appendix A.1.

$$
E\left\{\left[y^{2} \exp (y / \sigma)\right] /\left[1+\exp (y / \sigma)^{2}\right]\right\}=(2 / \sigma) \int_{0}^{\infty} \frac{y^{2} \exp (2 y / \sigma)}{[1+\exp (y / \sigma)]^{4}} d y .
$$

The evaluation of this integral is tedious, but can be accomplished by using the change of variable, $z=\exp (y / \sigma)$, and then repeatedly integrating by parts and by partial fractions. A final change of variable, $w=z^{-1}$, and the use of the integral $\int_{0}^{1} \frac{\ln (1+w)}{w} d w=\left(\pi^{2} / 12\right)$ (Gradshteyn, and Ryzhik, 1994; integral 4.291, no.1.), yields the result:

$$
E\left\{\left[y^{2} \exp (y / \sigma)\right] /[1+\exp (y / \sigma)]^{2}\right\}=\left(\sigma^{2} / 3\right)\left[\left(\pi^{2} / 6\right)-1\right] \text {. }
$$

$$
\begin{aligned}
& E\left\{\left[y^{3}(\exp (y / \sigma)-\exp (2 y / \sigma))\right] /[1+\exp (y / \sigma)]^{3}\right\} \\
& =(2 / \sigma) \int_{0}^{\infty} \frac{y^{3}[\exp (2 y / \sigma)-\exp (3 y / \sigma)]}{[1+\exp (y / \sigma)]^{5}} d y .
\end{aligned}
$$

This integral can be evaluated by using the same approach as in (ii), yielding:

$$
E\left\{\left[y^{3}(\exp (y / \sigma)-\exp (2 y / \sigma))\right] /[1+\exp (y / \sigma)]^{3}\right\}=\sigma^{3}\left[0.5-\left(\pi^{2} / 12\right)\right] .
$$




\section{References}

Adatia, A. (1997). Approximate BLUE's of the parameters of the half logistic distribution based on fairly large doubly censored samples. Computational Statistics and Data Analysis 24: 179-191.

Adatia, A. (2000). Estimation of the parameters of the half-logistic distribution using generalized rank set sampling. Computational Statistics and Data Analysis 33: 1-13.

Balakrishnan, N. (1985). Order statistics from the half logistic distribution. Journal of Statistical Computation and Simulation 20: 287-309.

Balakrishnan, N., Asgharzadeh, A. (2005). Inference for the scaled half-logistic distribution based on progressively type-II censored samples. Communications in Statistics - Theory and Methods 34: 73-87.

Balakrishnan, N., Chan, P. S. (1992). Estimation for the scaled half logistic distribution under Type II censoring. Computational Statistics and Data Analysis 13: 123-131.

Balakrishnan, N., Puthenpura, S. (1986). Best linear unbiased estimators of location and scale parameters of the half logistic distribution. Journal Statistical Computation and Simulation 25: 193-204.

Balakrishnan, N., Wong, K. H. T. (1991). Approximate MLEs for the location \& scale parameters of the half-logistic distribution with type-II censoring. IEEE Transactions on Reliability 40: 140-145.

Bartlett, M. S. (1953a). Approximate confidence intervals. Biometrika 40: 12-19.

Bartlett, M. S. (1953b). Approximate confidence intervals II. More than one unknown parameter. Biometrika 40: 306-317.

Cordeiro, G. M., Klein, R. (1994). Bias correction in ARMA models. Statistics and Probability Letters 19: 169-176.

Cox, D. R., Snell, E. J. (1968). A general definition of residuals. Journal of the Royal Statistical Society, B 30: 248-275.

Cribari-Neto, F., Vasconcellos, K. L. P. (2002). Nearly unbiased maximum likelihood estimation for the beta distribution. Journal of Statistical Computation and Simulation 72: 107-118.

Efron, B. (1982). The Jackknife, the Bootstrap and Other Resampling Plans. Philadelphia, PA: Society for Industrial and Applied Mathematics.

Gradshteyn, I. S., Ryzhik, I. W. (1994). Table of Integrals, Series, and Products (ed. A. Jeffrey), $5^{\text {th }}$ ed. New York: Academic Press. 
Haldane, J. B. S. (1953). The estimation of two parameters from a sample. Sankhyā 12: 313320.

Haldane, J. B. S., Smith, S. M. (1956). The sampling distribution of a maximum likelihood estimate. Biometrika 43: 96-103.

Kantam, R. R. L., Rosaiah, K. (1998). Half logistic distribution in acceptance sampling based on life tests. IAPQR Transactions 23: 117-125.

Maplesoft (2005), Maple 10. Waterloo, ON: Waterloo Maple.

Mbah, A. K., Tsokos, C. P. (2008). Record values from half logistics and inverse Weibull probability distribution functions. Neural, Parallel and Scientific Computations 16: 7392.

R (2008). The R Project for Statistical Computing, http://www.r-project.org

Shenton, L. R., Bowman, K. (1963). Higher moments of a maximum-likelihood estimate. Journal of the Royal Statistical Society, B 25: 305-317.

Shenton, L. R., Wallington, P. A. (1962). The bias of moment estimators with an application to the negative binomial distribution. Biometrika 49: 193-204.

Toomet, O., Henningsen, A. (2008). maxLik: Maximum likelihood estimation. http://CRAN.R-project.org; http://maxLik.org

Tukey, J. W. (1949). Standard confidence points. Memorandum Report No. 26, Statistical Research Group, Stanford University. 